\title{
Settling velocity and preferential concentration of heavy particles under two-way coupling effects in homogeneous turbulence
}

\author{
R. Monchaux \\ IMSIA, ENSTA-ParisTech/CNRS/CEA/EDF, Université Paris Saclay, \\ 828 Boulevard des Maréchaux, 91762 Palaiseau Cedex, France \\ A. Dejoan \\ Unidad de Modelización y Simulación de Procesos, \\ Centro de Investigaciónes Energéticas Medioambientales y Tecnológicas (CIEMAT), \\ Av. Complutense, 28040, Madrid, Spain
}

(Dated: September 6, 2017)

\begin{abstract}
The settling velocity of inertial particles falling in homogeneous turbulence is investigated by making use of Direct Numerical Simulation (DNS) at moderate Reynolds number that include momentum exchange between both phases (two-way coupling approach). Effects of particle volume fraction, particle inertia and gravity are presented for flow and particle parameters similar to the experiments of Aliseda et al. [1]. A good agreement is obtained between the DNS and the experiments for the settling velocity statistics, when overall averaged but as well when conditioned on the local particle concentration. Both DNS and experiments show that the settling velocity further increases with increasing volume fraction and local concentration. At the considered particle loading the effects of two-way coupling is negligible on the mean statistics of turbulence. Nevertheless, the DNS results show that fluid quantities are locally altered by the particles. In particular, the conditional average on the local particle concentration of the slip-velocity shows that the main contribution to the settling enhancement results from the increase of the fluid velocity surrounding the particles along the gravitational direction induced by the collective particle back-reaction force. Particles and the surrounding fluid are observed to fall together, which in turn results in an amplification of the sampling of particles in the downward fluid motion. Effects of two-way coupling on preferential concentration are also reported. Increase of both volume fraction and gravity is shown to lower preferential concentration of small inertia particles while a reverse tendency is observed for large inertia particles. This behavior is found to be related to an attenuation of the centrifuge effects and to an increase of particle accumulation along gravity direction, as particle loading and gravity become large.
\end{abstract}

\section{INTRODUCTION}

Numerical and experimental studies [1-6] have shown that heavy particles falling in homogeneous isotropic turbulence settle faster than in a quiescent fluid. This phenomena is explained by the inertial bias mechanism, responsible of the migration of heavy particles to the periphery of turbulent vortical structures in zero-gravity conditions and which, under gravity effects, preferentially sample the side of descending fluid motions of such structures. The understanding of the mechanisms underlying preferential concentration under gravitational acceleration is thus central to the settling problem. Some examples of its relevance in natural and industrial flows are as diverse as aerosol transport in the atmosphere or mixing of sprays in combustors. In a previous study [7] we examined the preferential concentration and settling issues by making use of the Voronoï diagrams method to analyze data extracted from Eulerian-Lagrangian Direct Numerical Simulation (DNS) that do not include the effect of particles on the carrier fluid (referred to as "one-way coupling simulation approach"). In agreement with earlier findings, both preferential concentration and settling enhancement were found maximum when the particles have a response time $\tau_{p}$ close to the Kolmogorov time scale $\tau_{\eta}$, and the statistics of the particle velocity conditioned on the local concentration showed a clear correlation between the particle accumulation and the increase of the falling velocity. In addition, we identified a further contribution to the settling increase due to a preferential sampling by the particles of regions of descending fluid acceleration (beside the preferential sampling by particles of regions of descending velocity, referred to as "preferential sweeping" by Wang \& Maxey [2]).

In the present study we address the preferential concentration and particle settling mechanisms by including back-reaction force of the particles on the carrier flow in our DNS -the so-called "two-way coupling" simulation approach. Two-way coupling simulations are usually used to study the alteration of mean turbulence statistics by the presence of the particles in zero-gravity environment [8-11] and marginally in presence of gravity $[5,12,13]$. The experiments [1] and numerical simulations on particles settling in turbulence $[4,14]$ have mainly focused on the particle mean statistics and shown that two-way coupling further increases the falling velocity. Here, we propose a local analysis of gravitational settling under two-way coupling based on both particle and fluid statistics conditioned on the local particle concentration. Our main objective is to get further insights into the local interplay between preferential concentration and turbulence, and the resulting effects on the par- 
ticle settling. Effects of volume fraction $\Phi$, Stokes number $S t=\tau_{p} / \tau_{\eta}$ and Rouse number $R$ (being defined as $R=v_{t} / u^{\prime}$, the ratio of the terminal velocity of the particle $v_{t}$ to the turbulence intensity $u^{\prime}$ ) are examined.

The paper is organized as follows. First, we describe in Sec. II the numerical simulations and the post-processing of flow and particle data. In Sec. III we analyze effects of particle inertia, Rouse number and particle volume fraction on settling velocity, preferential concentration and fluid quantities. This includes mean and conditional statistics for particle and fluid fields. Finally, Sec. IV provides a discussion of the presented results by highlighting the most relevant conclusions.

\section{NUMERICAL SIMULATIONS AND POST-PROCESSING}

\section{A. Numerical simulations}

The homogeneous and isotropic turbulence is described in the Eulerian reference frame by the incompressible Navier-Stokes equations:

$$
\frac{\partial u_{i}}{\partial t}+\frac{\partial u_{j} u_{i}}{\partial x_{j}}=-\frac{1}{\rho} \frac{\partial p}{\partial x_{i}}+\nu \frac{\partial^{2} u_{i}}{\partial x_{j} \partial x_{j}}+f_{i}-f_{i}^{(p)}+F_{i}^{0},
$$

where $i=1,2,3$ refers to the three Cartesian directions (gravity is along the third one denoted $z$ in the following), $x_{i}$ is the spatial coordinates, $u_{i}$ the fluid velocity, $p$ the pressure and $\rho$ and $\nu$ the fluid density and kinematic viscosity. The statistically steady turbulence is achieved through the external energy source term, $f_{i}$, that injects energy at low wavenumbers such that turbulence energy dissipation is balanced. Also, to avoid further acceleration of the particles induced by non-zero net volume flux along the gravity direction $[4,15]$, the mean flow (integrated over the computational domain) is imposed to be zero. This is equivalent to apply a constant mean pressure gradient, $F_{i}^{0}$, that balances the net weight of the particle phase - see Eq. (1) - and below for its definition.

The term $-f_{i}^{(p)}$ represents the force per unit mass exerted by a number of $n_{p}$ particles within the integration control fluid volume $v_{\text {cell }}$ and is computed according to the Particle In Cell (PIC) method $[4,12,13,16]$ :

$$
f_{i}^{(p)}=\frac{1}{m_{v_{\text {cell }}}} \sum_{j=1}^{n_{p}} f_{i}\left(p_{j}\right),
$$

where $m_{v_{\text {cell }}}$ is the mass of fluid within the integration control volume and $f_{i}\left(p_{j}\right)$ is the drag force acting on the particle $p_{j}$ in the $i$-direction (see Eq. 3).

The particles, with density $\rho_{p}$ much larger than the fluid density $\rho$, are described in the Lagrangian reference frame by a simplified version of the equation of motion of Maxey, Riley and Gatignol $[17,18]$ where only the Stokes drag and buoyancy forces remain:

$$
\begin{aligned}
m_{p_{j}} & \frac{d v_{i}\left(x_{p_{j}}, t\right)}{d t}= \\
& \underbrace{m_{p_{j}} \frac{\left(u_{i}\left(x_{p_{j}}, t\right)-v_{i}\left(x_{p_{j}}, t\right)\right)}{\tau_{p}}}_{f_{i}\left(p_{j}\right)}+\left(m_{p_{j}}-m\right) g_{i},
\end{aligned}
$$

where $v_{i}(i=1,2,3)$ are the particle velocity components, $u_{x_{p_{i}}}$ the instantaneous fluid velocity at the particle location $x_{p_{j}}, m_{p_{j}}$ the particle mass and $m$ the mass of fluid one particle displaces. The response time of the particles, $\tau_{p}$, is given by $\tau_{p}=d^{2} \rho_{p} /(18 \nu \rho)$ with $d$ being the particle diameter. The gravitational acceleration $g_{i}$ is such that $g_{1}=g_{2}=0$ and $g_{3}=-|g| x_{3}$ where $|g|$ and satisfies $v_{t}=\tau_{p}|g|\left(1-\rho / \rho_{p}\right), v_{t}$ being the terminal velocity of the particles in the still fluid. Note that, for a prescribed value of the Reynolds and Rouse numbers, the effects of gravity and inertia can be tuned independently. Indeed, these effects can be analysed for a given Froude number $F r=a_{o}^{1 / 2}\left(\eta / t_{\eta}^{2}\right) /|g|$ (where $a_{o}^{1 / 2}\left(\eta / t_{\eta}^{2}\right)$ is the fluid acceleration variance and is close to unity in our DNS), or for a given particle response time $\tau_{p}$.

The Navier-Stokes equations are solved on a cubic fluid box of side length $L_{\text {box }}=2 \pi$, discretized into $N^{3}$ computational nodes, with periodic boundary conditions. A fully pseudo-spectral algorithm with a de-aliasing truncation technique (referred to as the " $2 / 3$ rule") is used with a second-order Runge-Kutta time-stepping for the non-linear terms and an analytic integrating factor for the viscous terms. The forcing is realized by distributing the power input $f_{i}$ over a narrow band of wavenumbers $k$ that satisfy $k_{p}-1 \leq k \leq k_{p}+1$, where $k_{p}$ defines the peak forcing mode (see $[19,20]$ for further computational details). Note that the above-mentioned condition of a net zero-volume flux along gravity, leads to $F_{i}^{0}=-\left(\rho_{p} / \rho-1\right) g_{i} \times N_{p} V_{p} / L_{\text {box }}$ where $N_{p}$ is the total number of particles and $V_{p}$ the particle volume.

A fourth-order Lagrangian polynomial interpolation is used to evaluate the fluid velocity at the particle position required for the computation of the drag force exerted by the fluid on the particle, $f_{i}\left(p_{j}\right)$. The particles are homogeneously introduced in the fluid once the turbulence shows a statistically stationary state and the statistics of the particle fields are initiated over several integral time scales $\left(\sim 20 T_{o}\right)$ after their injection.

The numerical and turbulence parameters for the unladen flow are identical to the ones used in our previous study [7] and recalled in Table I. Table II provides the values of the Stokes number $S t$, Rouse number $R$, total number of particles $N_{p}$ and volume fraction $\Phi=N_{p} V_{p} / L_{\text {box }}^{3}$, considered in the two-way coupling simulations. This table provides the parameter values based on prescribed Stokes numbers (i.e. gravity varying according to $R$ ), but the main reported characteristics are similar to the ones found for a given Froude number (i.e particle inertia varying according to $R$ ). In all simulation cases, $N_{p}$ refers to real particles and not to compu- 
tational particles [13], and $\Phi$ is small enough to discard "four-way coupling" (particle/particle interactions) [21]. All the particles have a density $\rho_{p}=5000 \rho$. Whatever the parameter values we are considering, the computational domain is large enough to avoid artificial periodic boundary condition effects (see Woittiez et al. [22]). This is illustrated in Fig. 1 which represents an instantaneous view of the particle position in a $2 \mathrm{D}$ plane containing gravity for $S t=1, \Phi=7 \times 10^{-5}$ and $R=1$. Large scale particle clusters crossing the full computational domain are not observed.

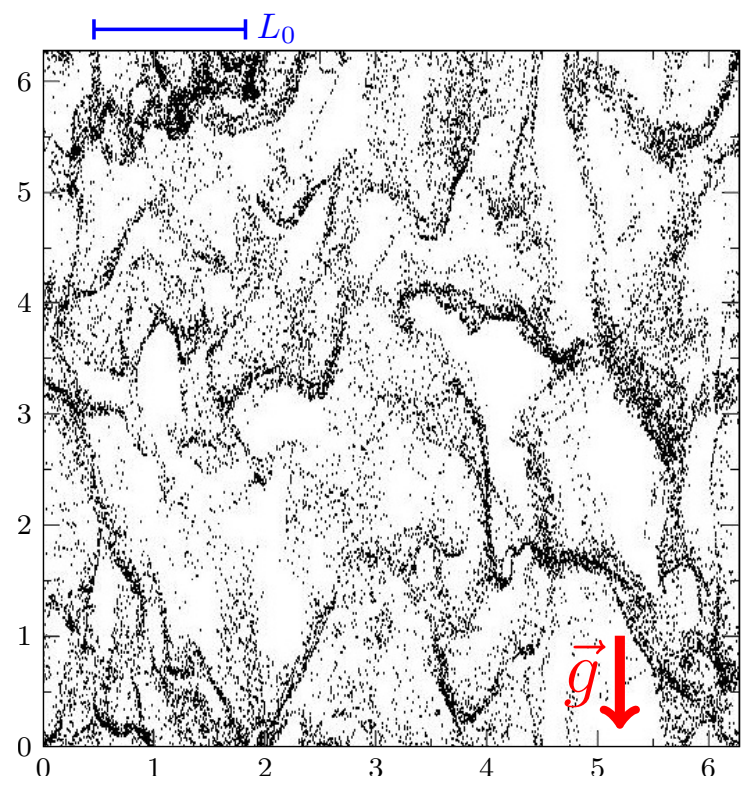

FIG. 1. Particle position in a $2 \mathrm{D}$ pane containing gravity for $S t=1, \Phi=7 \times 10^{-5}$ and $R=1$. The red arrow reprentents the gravity vector and the blue line is the integral length scale $L_{o}$.

\begin{tabular}{lcccccc}
\hline$R_{\lambda} N^{3}$ & $\nu$ & $L_{\circ} / L_{\text {box }}$ & $L_{o} / \eta_{o}$ & $T_{o} / t_{\eta_{o}}$ & $k_{\max } \eta_{o}$ \\
\hline 40 & $64^{3}$ & 0.0178 & 0.211 & 31.56 & 15.85 & 1.32 \\
\hline
\end{tabular}

TABLE I. Unladen turbulence: numerical and flow parameters. Microscale Reynolds number $R_{\lambda}$, number of computational nodes $N^{3}$, viscosity $\nu$, box side length $L_{\text {box }}$, integral length scale $L_{o}$, large-eddy turn-over time $T_{o}$, Kolmogorov length and time scales $\eta_{o}$ and $t_{\eta_{o}}$ and maximum wavenumber $k_{\max }=\sqrt{2} N / 3$

Table II includes some ratio of particle-laden to unladen turbulence mean quantities to supply an overview of the flow modulation by the particles. For the volume fractions considered, $\Phi=\mathcal{O}\left(10^{-5}\right)$, the mean statistics of turbulence are not significantly altered by the particles, the stronger modulation being observed for large $R$ and $\Phi$. The slight tendency indicated by the values reported in Table II is in agreement with previous studies on modification of homogeneous turbulence by two-way

\begin{tabular}{c|c|c|c|c|c|c}
$S t_{o}$ & $\begin{array}{c}R_{o} \\
\left(v_{t} / u^{\prime}\right)\end{array}$ & $10^{5} \Phi$ & $N_{p}$ & $\begin{array}{c}u^{\prime} / u_{o}^{\prime} \\
\left.\left(R_{o} / R\right)\right)\end{array}$ & $\begin{array}{c}\eta / \eta_{o} \\
\left(\varepsilon_{o} / \varepsilon\right)\end{array}$ & $\begin{array}{c}S t / S t_{o} \\
\left(t_{\eta_{o}} / t_{\eta}\right)\end{array}$ \\
\hline 1 & 0 & 1.5 & 446080 & 0.99 & 1 & 1 \\
1 & 0 & 3 & 892032 & 0.97 & 0.98 & 1 \\
1 & 0 & 7 & 2083200 & 0.95 & 0.96 & 1 \\
\hline 1 & 0.25 & 1.5 & 446080 & 0.99 & 1 & 1 \\
1 & 0.25 & 3 & 892032 & 1 & 0.98 & 1 \\
1 & 0.25 & 7 & 2083200 & 1 & 0.88 & 1.25 \\
\hline 1 & 0.5 & 1.5 & 446080 & 1 & 0.97 & 1 \\
1 & 0.5 & 3 & 892023 & 1 & 0.9 & 1.2 \\
1 & 0.5 & 7 & 2083200 & 1.30 & 0.8 & 1.53 \\
\hline 1 & 1 & 1.5 & 446080 & 1.13 & 0.90 & 1.2 \\
1 & 1 & 3 & 892032 & 1.35 & 0.71 & 1.57 \\
1 & 1 & 7 & 2083200 & 1.72 & 0.64 & 2.64 \\
\hline 0.36 & 0.25 & 1.5 & 2060800 & 0.98 & 0.95 & 1 \\
0.36 & 0.25 & 3 & 4121600 & 1 & 0.95 & 1.13 \\
0.36 & 0.25 & 7 & 9617024 & 1.2 & 0.85 & 1.38 \\
\hline 2 & 0.25 & 1.5 & 154880 & 0.99 & 1 & 0.99 \\
2 & 0.25 & 3 & 309760 & 0.95 & 1 & 0.9 \\
2 & 0.25 & 7 & 722752 & 0.94 & 0.97 & 1 \\
\hline 6 & 0.25 & 1.5 & 29762 & 0.97 & 1 & 0.94 \\
6 & 0.25 & 3 & 59616 & 0.95 & 1 & 0.9 \\
6 & 0.25 & 7 & 139104 & 0.93 & 1.1 & 0.83 \\
\hline
\end{tabular}

TABLE II. Particle-laden turbulence: particle and turbulence parameters. Stokes number $S t$, volume fraction $\Phi$, total number of particles $N_{p}$. See Table I for definition of the turbulence parameter. Parameters with underscript "o" refer to the unladen flow. The error of the reported statistics does not exceed $2 \%$.

coupling effects (see details in $[5,9,23]$ for zero-gravity and $[12-14,24]$ for non zero-gravity condition). We recall that the present numerical study aims to investigate local two-way coupling effects to get further insight into the mechanisms underlying the increase of particle settling velocity, discarding overall two-way coupling effects on turbulence, in the prolongation of the experimental work of Aliseda et al. [1]. The moderate Reynolds number, $R_{\lambda}=40$, we consider in our simulations matches the one examined in [1], allowing direct comparisons between our DNS and the experiments. It also permits to explore a sufficient large range of particle parameters to extract relevant effects of Stokes and Rouse numbers and of volume fraction, while consuming reasonable computational memory and CPU times. The considered particle parameters here correspond to particles with diameter smaller than the Kolmogorov length scale $\eta$. Taking as reference $\eta=1 \mathrm{~mm}$ for the atmospheric boundary layer, they can be representative of droplets with diameter in the range [0.08-0.3 mm] or very small heavy aerosols with diameters in the range [0.03-0.1 mm].

\section{B. Post-processing}

To analyze, locally, the interplay between settling enhancement and preferential concentration of particles, the computation of the concentration field at each particle location is required. This can be efficiently achieved 
by making use of the Voronoï diagrams analysis [25]. This method associates to each particle a unique polyhedron, defined as the subset of the $3 D$ space that is closer to this particle than to any other. According to this definition, the corresponding polyhedron volume is exactly the inverse of the local concentration defined at the intrinsic inter-particle distance scale. The mean Voronoï volume associated to $N_{p}$ particles being trivially equal to $L_{\text {box }}^{3} / N_{p}$, the Voronoï volumes are normalized to achieve unit mean and are denoted by $\mathcal{V}$. Note that a quantitative comparison between different sample sizes is possible as long as the statistical post-treatment is performed over data sets presenting similar average of the inter-particle distance. This condition is carried out by applying the sub-sampling procedure fully described in [26] and more detailed below in Sec.III B.

Following Monchaux et al. [27], we define a cluster of particles as connected components of Voronoï cells whose individual volume is below a given threshold. This threshold can be simply defined as the intersection of the preferentially concentrated particle PDF of $\mathcal{V}$ with the corresponding uniform random distribution PDF.

For each simulation case, the statistical analysis is performed over 20 snapshots regularly sampling 8 integral time scales (after statistical stationary convergence is achieved). The overall average operator denoted by an over-bar, $\bar{X}$, represents the average operator over time and space of the quantity $X$, and its associated standard deviation is denoted as $\sigma_{X}$. The local concentration, $C$, measured as the inverse of the normalized Voronoï volume is used to perform the statistical analysis conditioned on the local concentration. By reference to the average concentration, $C_{0}=N_{p} /(2 \pi)^{3}$, we define six ranges of relative concentration: $C / C_{0} \ll 1, C / C_{0} \simeq 0.3$, $C / C_{0} \simeq 0.7, C / C_{0} \simeq 1.3, C / C_{0} \simeq 2.2$ and $C / C_{0} \simeq 6.7$.

\section{RESULTS}

\section{A. Settling velocity}

Figure 2(a) displays the mean rate of particle settling velocity as a function of $R$. It provides a comparison between our two-way and one-way coupling DNS, the experiments of Aliseda et al. [1], performed at a low Reynolds number, and with the experiment and one-way coupling DNS results of Good et al. [6], performed at a much higher Reynolds number. All data correspond to Froude numbers close to unity and to low volume fractions $\left(\Phi \leq 1.5 \times 10^{-5}\right)$ for which two-way coupling effects are weak. It is interesting to observe that experimental data of Aliseda et al. and Good et al. exhibit very similar behaviors though $R_{\lambda}$ varies from 46 to 160 . Similarly data from both one-way coupling DNS performed at different Reynolds numbers, collapse for $R \leq 0.3$, where Stokes numbers correspond to particles interacting with the small turbulence scales. At larger $R$, the settling rate obtained in the low Reynolds number DNS is smaller than the one found at high Reynolds numbers, nevertheless, the decay slope of the settling velocity is similar for both simulations. For a given Froude number, increasing $R$ is equivalent to increasing particle inertia. The interaction between particles and turbulence is thus expected to be more subject to Reynolds number effects at large $R$ since the range of turbulence scales interacting with large particle inertia extends more as $R_{\lambda}$ increases. The behavior of the settling rate displayed by our two-way coupling DNS is comparable to the one observed in the experimental work [1]: an increase of the settling velocity up to $R \simeq 0.4$ and then a decay for larger $R$. The value of $R$ at which maximum of settling velocity increase occurs and the decay rate for larger $R$ compares well. The experimental enhancement of the settling rate for small $R$ is also well captured by our DNS. However, the absolute values are underestimated, a feature shared by all the presented DNS data when compared with corresponding experiments. Comparison between our one-way and two-way coupling DNSs shows that even for the smallest volume fraction, $\Phi=1.5 \times 10^{-5}$, momentum exchange between particles and fluid enhances the settling rate. The effects of volume fraction effects will be further discussed below. Note that the largest values of $R$ explored in our two-way coupling DNS and in the experiment of Aliseda et al. is around unity. Thus, the hindering effect (reduction of the settling velocity) found by Good et al. at large $R$ cannot be recovered. Further investigation of this phenomena requires a supplementary exploration of the flow and particle parameters and is not considered in the present work.

Figure 2(b) displays the mean rate of particle settling velocity as a function of $S t$ for the present two-way coupling DNS and Aliseda et al. experiments. For the DNS, we draw both the results obtained for $F r=1$ (gravity is fixed and $R$ varies with particle inertia as in Fig. $2(\mathrm{a})$ ) and for $R=0.25$ ( $F r$ varies with particle inertia). For both experiments and two-way coupling DNS, two volume fractions are considered, $\Phi=1.5 \times 10^{-5}$ (open symbols) and $\Phi=7 \times 10^{-5}$ (filled symbols). All data sets show a maximum increase of the settling velocity reached for $S t$ around unity. This maximum, in agreement with previous numerical and experimental studies $[1-3,5,7]$, is consistent with the peak of settling rate observed in figure 2 (a) for $R \sim 0.4$, which corresponds to $S t \sim 1.5$ in our DNS. This peak was first explained in one-way coupling DNS by Wang \& Maxey [2] as being the result of both preferential concentration (due to centrifuge effects that are effective on particles with time response close to the Kolmogorov time scale) and preferential sweeping (preferential aglomeration of particles in descendant fluid region under gravity). The alteration of these mechanisms by two-way coupling effects will be more discussed in Secs. III B and III C.

Also shown in Fig.2(b) is that, at the low $\Phi=1.5 \times$ $10^{-5}$, the DNS results do no exhibit significant influence of the Froude number on the mean settling rate and both sets of DNS data (with $F r=1$ or $R=0.25$ ) are close 

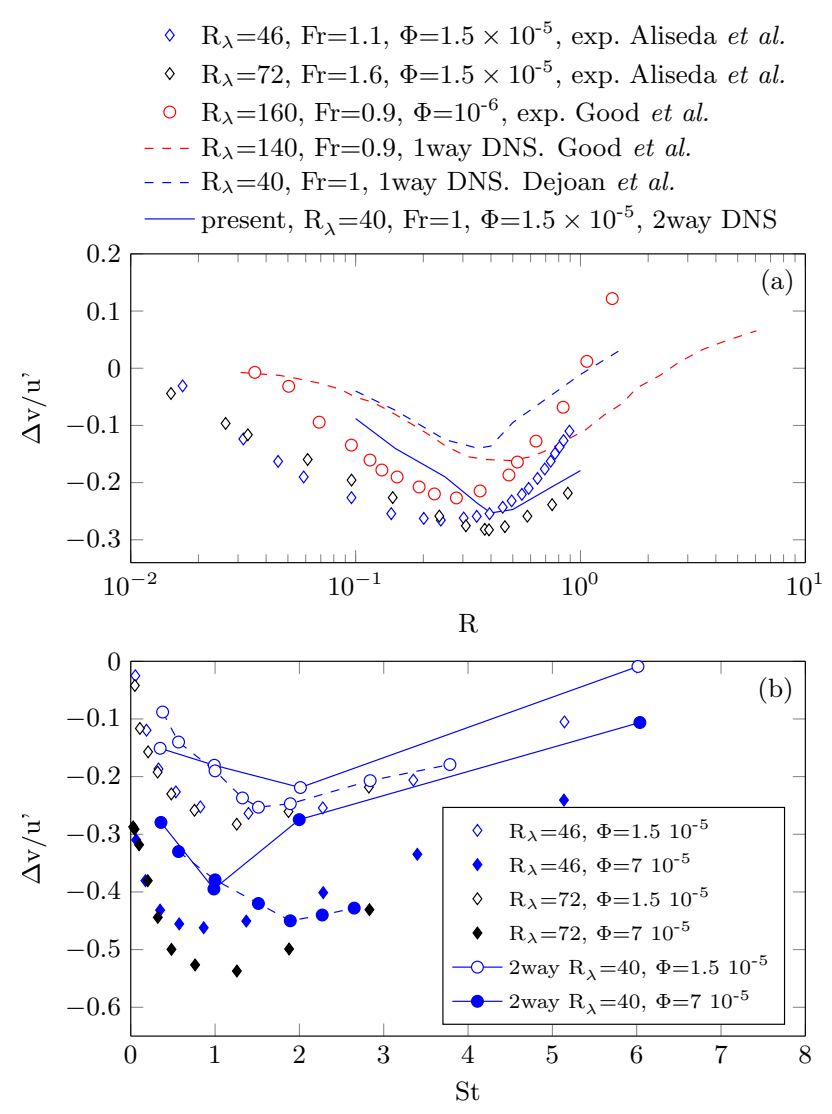

FIG. 2. Color online. Rate of the settling velocity $\Delta v=$ $\overline{v_{z}-v_{t}}$ normalized by $u^{\prime}$, for low $\Phi$ : (a) as a function of $R$ for $F r \simeq 1$, (b) as a function of St. Data from Aliseda et al. [1], from Good et al. [6] and from our one-way study [7] are given for comparison. In (b) solid lines represent the DNS performed with $R=0.25$ (corresponding to $0.38<F r<6.1$ for the prescribed range of $S t$ values) while dashed lines the DNS performed with $F r \sim 1$ (corresponding to $0.1<R<1$ for the prescribed range of $S t$ values). Data at $R_{\text {lambda }}=46$ and $R_{\lambda}=72$ are experiments by Aliseda et al. [1].

to the experimental data. For $\Phi=7 \times 10^{-5}$, the DNSs show that effects of $F r$ are very weak for small $S t$ but become large for $S t \geq 1$. In this range of particle inertia, the DNS recovers better the data of Aliseda et al. when $F r$ is similar to the experimental one, i.e. $F r=1$. Note that the tendency of the DNS in underestimating the settling rate for small $S t$ is reminiscent of the previously and similar trend observed in Fig. 2(a) for small $R$.

Figure 3 shows that, for a given $R$ or a given $S t$, the mean settling rate velocity increases with the volume fraction both in our two-way coupling DNS and in experiments. By comparing Figs. 3(a) and 3(b), it appears that influence of $R$ is much weaker than that of $S t$. The correlation between particle concentration and settling velocity is further illustrated in Fig. 4 that displays the evolution of the settling velocity deviation from its mean value with the local relative concentration $C / C_{0}$. When the local relative concentration is higher than about 2.5,

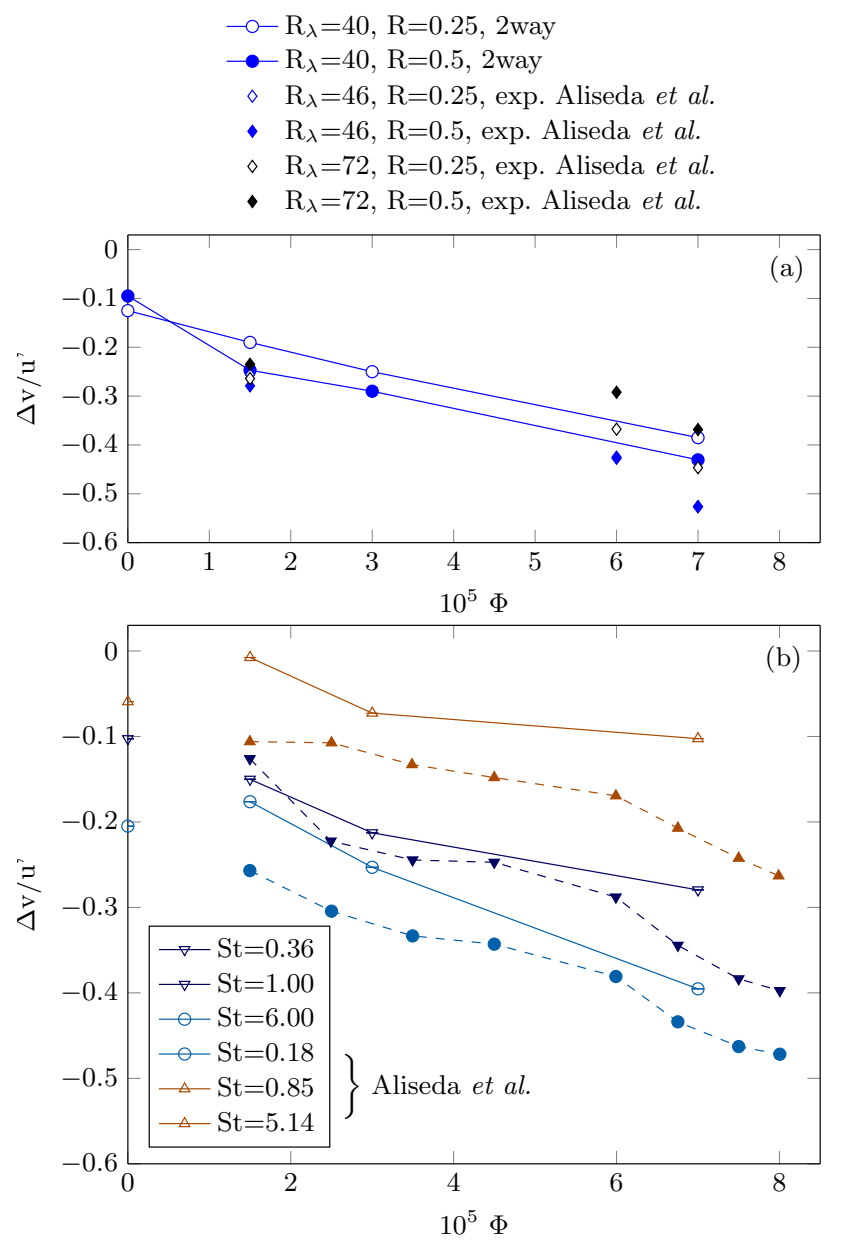

FIG. 3. Color online. Rate of the settling velocity $\Delta v=$ $\overline{v_{z}-v_{t}}$ normalized by $u^{\prime}$ as a function of $\Phi$. (a) for prescribed values of $R$ (with $F r \sim 1$ ). (b) for prescribed values of $S t$. Continuous lines correspond to our two-way coupling data at $R=0.25$, dash-dotted lines/symbols to data extracted from Aliseda et al. [1] for corresponding values of $\Phi$. Note that $\Phi=0$ represents the corresponding one-way coupling DNS data.

particles settle faster than on average and the opposite is observed when $C / C_{0} \leq 2.5$. Obviously, averaged over $C / C_{0}$, all the data reduce to zero, and the main relevance from one curve to another one is how much the relative particle settling is increased at high local concentration. The present results, shown for $S t=1$ and $R=0.25$, are compared with our one-way coupling DNS [7] and the experimental data [1]. At high local concentrations, the two-way coupling simulations exhibit a larger slope of settling enhancement than one-way coupling simulations. The slope increases as $\Phi$ becomes larger and quantitatively matches the data of Aliseda et al. for $\Phi=7 \times 10^{-5}$.

Alteration of preferential concentration and relevant fluid quantities by two-way coupling effects are next analysed to get further insight in the additional increase 


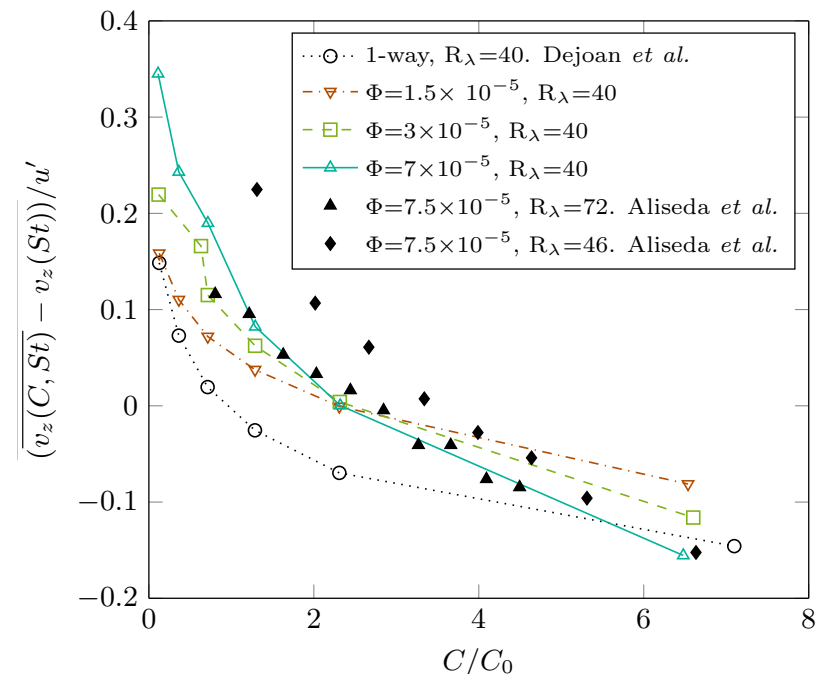

FIG. 4. Color online. Conditional average of settling velocity enhancement given as a function of the local concentration $C / C_{0}$ for $S t=1, R=0.25$ and various values of $\Phi$. For comparison, our 1-way coupling DNS work [7] and data from Aliseda et al. [1] are included.

of settling velocity when compared to one-way coupling DNS.

\section{B. Preferential concentration and clustering}

As briefly mentioned in Sec. IIB, consistent comparisons between statistics of the Voronoï diagrams performed over different data sample, require to maintain as close as possible the inter-particle distance, $\Delta d_{p}=$ $L_{\text {box }} / N_{p}^{1 / 3}$, of the data sets considered for the statistical post-treatment. This is illustrated in Fig. 5(a) that displays the evolution of $\sigma_{\mathcal{V}}$ as a function of $S t$ (for $R=0.5$ and the three considered $\Phi$ ) computed from two different present DNS data sample sizes. The sub-sampling procedure [26] was applied to the each simulation particle data set by selecting as the reference sample the one corresponding to our previous one-way coupling DNS, that contains a total number of 320000 particles (or equivalently with $\Delta d_{p} \sim 0.09$ ). Moreover, we have used the relation of proportionality of $\sigma_{\mathcal{V}}$ with $\Delta d_{p}$ demonstrated in [26] to extrapolate the values of $\sigma_{\mathcal{V}}$ for the sample size smaller (29762 particles) than the reference case. Note that this sub-sampling procedure has also been recently used by Sumbekova et al. [28]).

The maximal preferential concentration for $S t \simeq 2 \mathrm{ob}-$ served in Fig. 5 has been commonly reported in experiments [27] and in one-way coupling simulations [7, 26]. This maximum is shown to be independent of particle loading in the present simulations. By comparison to our former one-way coupling DNS, the level of preferential concentration tends to be lower for $S t \leq 2$ and higher for the larger particle inertia, $S t=6$. The dependence in
$R$ and $\Phi$ of $\sigma_{\mathcal{V}}$ presented in Fig. 5(b) for $S t=1$, shows a decrease of preferential concentration as both $R$ and $\Phi$ increase.
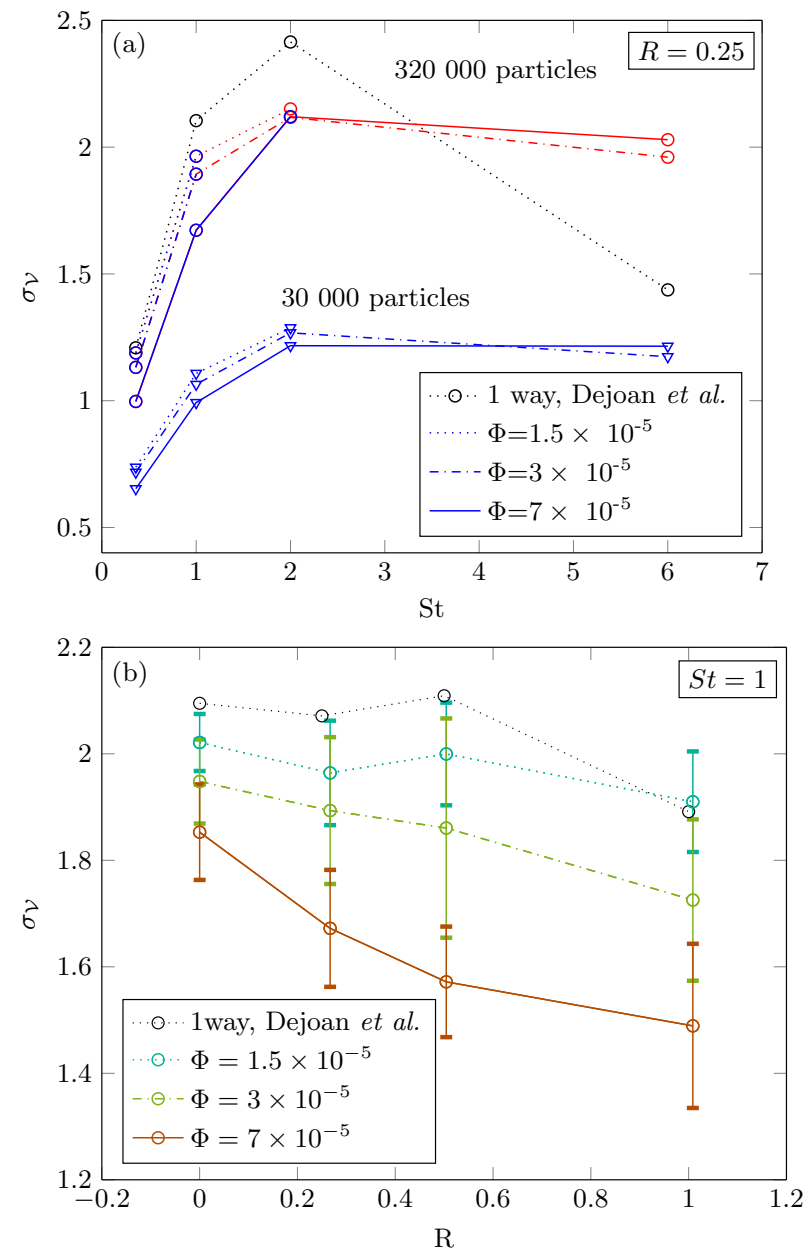

FIG. 5. Color online. Top: standard deviation of the normalized Voronoï volume as a function of $S t$ given for each volume fractions $\Phi$ considered at $R=0.25$. The two sets of curves correspond to the two sample sizes used (29762 and 320000 particles). Data presented in red (light gray) are extrapolated, see text for details. Bottom: standard deviation of the normalized Voronoï volume as a function of $R$ at $S t=1$ for each volume fractions $\Phi$ considered. 1-way data from Dejoan et al. [7] are given as reference.

We further analyze the two-way coupling effects on the particle concentration field by performing Voronoï analysis on 2D slices aligned with the axis of the simulation. We define three slice orientations, two of them contain gravity, the other one does not. We consider as well two quantities: on one hand, the aspect ratio of the $2 \mathrm{D}$ Voronoï cells defined as $\lambda_{1} / \lambda_{2}$ (with $\lambda_{1}$ and $\lambda_{2}$ the two principal moments of inertia of the considered cells) and, on the other hand, the angle between the longer axis of inertia of the Voronoï cell and the simulation box axis $\left(\overrightarrow{e_{x}}, \overrightarrow{e_{y}}, \overrightarrow{e_{z}}\right)$.

Not shown here, the probability density functions 
(PDFs) of $\lambda_{1} / \lambda_{2}$ show that the most probable shape of the Voronoï cells corresponds to elongated ellipses whose characteristics depend mainly on $S t$ (as already found in our previous one-way coupling DNS [7]) and are not signicantly influenced by $R$ and $\Phi$. However, Fig. 6 clearly shows that, as $R$ and $\Phi$ increase, the Voronoï cells tend to align perpendicular to gravity, the particles preferentially agglomerating along the falling direction than along the transverse directions. The increase of anisotropy of the particle field with increasing $R$, previously reported in our one-way coupling DNS study, corroborates more recent one-way coupling DNS results [29, 30]. In particular, the DNS by Ireland et al. [30] have shown that, as gravity is increased, particles with low $S t$ fall faster and thus pass through flow structures, leading to a lower degree of preferential concentration (the particles being less subject to centrifuge and preferential sweeping effects [2]); on the contrary, for particle with large $S t$, they observe an increase of clustering in relation with the reduction of the past-history effects along the downward direction. Indeed, the particles tend to concentrate along gravity instead of being more homogeneously dispersed. The present simulations show that momentum exchange between the phases further amplifies the preferential orientation of particles along gravity as $\Phi$ and/or $R$ increase. Following [30], this, in relation with the settling enhancement reported in Sec. III A, can explain the decrease of preferential concentration for $S t \leq 2$ and the reverse tendency observed for larger $S t$ in Fig. 5 . Nonetheless, the amplified alignment of particles along the downward direction found here, when compared to our one-way coupling DNS, is obviously a result of the back-reaction of the particles on the fluid. This aspect is further examined in Sec. III C.

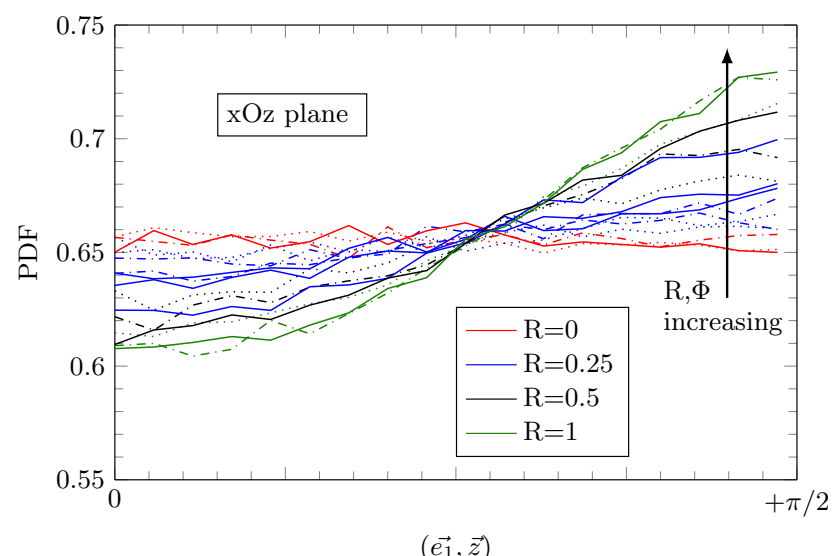

FIG. 6. Color online. PDFs of the angle between the main axis of the 2D Voronoï cells and the gravity direction for $S t=$ 1. Positive and negative angles have been averaged to increase convergence and improve readability. Corresponding PDFs in the plane perpendicular to gravity is perfectly flat (not shown).

As explained in Sec. II B, clusters can be identified as connected components of particles whose Voronoï cell has a volume below a given threshold. We have performed $2 \mathrm{D}$ and $3 \mathrm{D}$ analysis of the cluster size. For the $2 \mathrm{D}$ analysis, we have worked with slices aligned with the simulation axis as explained above. In this case, we find clusters whose sizes (areas) are algebraically distributed as power laws with exponents of about -2 as already reported by former numerical and experimental studies $[7,27,31]$. This is illustrated in Fig. 7 in which the cluster area distribution obtained for $\Phi=3 \times 10^{-5}$ and $S t=1$ in a plane containing gravity is presented. Cluster area PDFs also present a most probable value located in the range of turbulent scales $[2 \eta-4 \eta]$. This feature slightly differs from the one provided by the $2 \mathrm{D}$ cluster size analysis in the experiments of Aliseda et al. where box counting method pointed to cluster typical sizes distributed around $[7 \eta-16 \eta]$. Not explicitly shown, the cluster size distribution is found to be independent of $\Phi$, $R, S t$ and to not either depend on the orientation of the plane with respect to gravity. The independence with the Stokes number was already observed in experiments [27]. The $3 \mathrm{D}$ analysis reveals that, in presence of two-way coupling, the Voronoï cells are all interconnected. According to the definition of cluster we use, this interconnexion is representative of a single cluster with an entangled $3 \mathrm{D}$ structure that is reminiscent of the complex $3 \mathrm{D}$ interconnected tunnels of particles found by Calzavarini et al. making use of the Minkowski functionals [32] for analysing clustering in homogeneous turbulence.

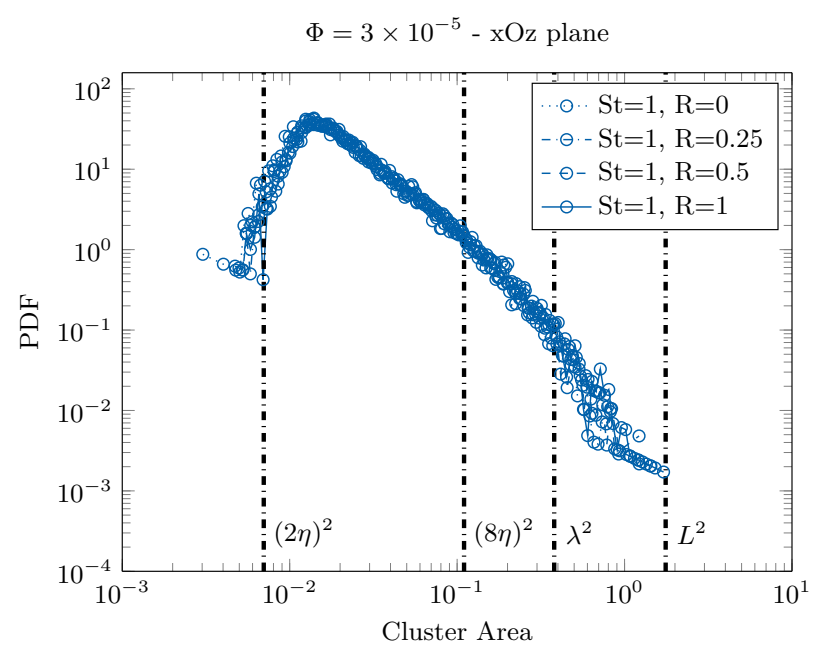

FIG. 7. Typical cluster size distribution in a $2 \mathrm{D}$ plane containing gravity for $S t=1$ and various values of $R$. Similar results are obtained for the other considered $S t$ values but are not shown for the sake of clarity.

\section{Fluid statistics at particle position}

A direct consequence of the model used for the computation of the Stokes drag (see Eq. 3), is the matching 

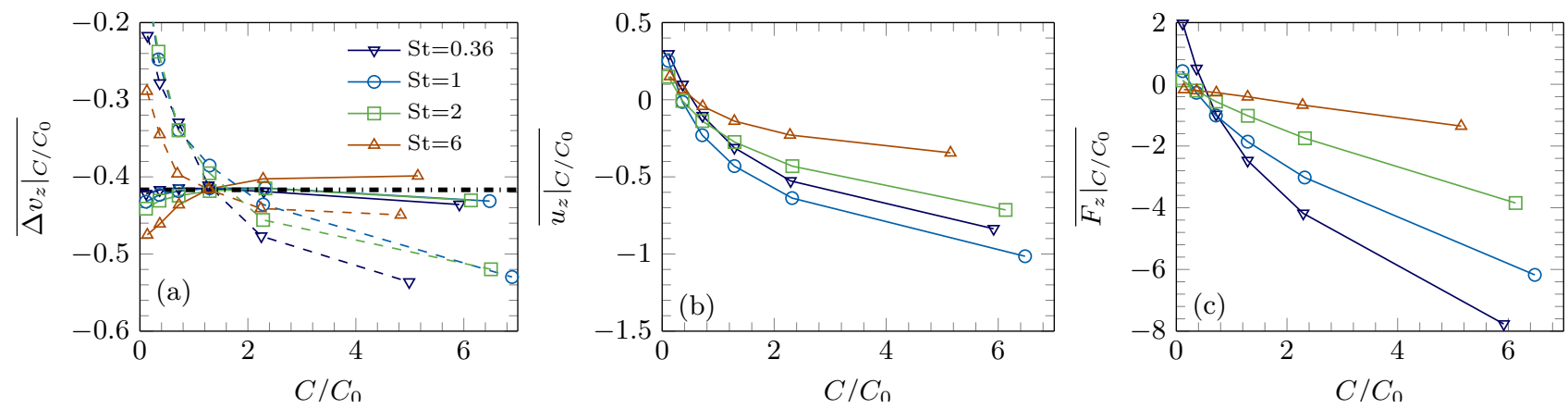

FIG. 8. Color online. Conditional average of the (a) slip velocity $\overline{\left.\Delta V_{z}\right|_{C / C_{0}}}$, (b) fluid velocity $\overline{\left.u_{z}\right|_{C / C_{0}}}$, and (c) net force exerted by the particles on the fluid $\overline{\left.F_{z}\right|_{C / C_{0}}}$, given as a function of the local concentration $C / C_{0}$ for $R=0.25$ and $\Phi=7 \times 10^{-5}$. In (a), the horizontal dot-dashed line is the terminal velocity $v_{t}$ and the dashed lines are the corresponding one-way coupling DNS slip velocities [7].

between mean average slip-velocity $\overline{\Delta v}_{z}$ and the terminal velocity $v_{t}$. Thus, the mean increase of the settling velocity comes from the local mean contribution of the fluid velocity. It is interesting to observe in Fig. 8(a) that, when considering conditional statistics on the local particle concentration, the slip-velocity remains equal to the terminal velocity regardless of the local concentration. This behavior differs from the one reported in our one-way coupling DNS study recalled in Fig. 8(a) by the dashed lines. Under one-way coupling, particles are observed to settle faster than the mean downward velocity in regions of high-local particle concentration, in relation with a preferential sampling along the fluid downward acceleration in addition to the preferential sweeping [2]. The matching between the conditional average of slipvelocity and terminal velocity found in two-way coupling (in regions of small and large particle density) indicates that the enhancement of the particle settling rate results essentially from the modified fluid velocity $\bar{u}_{z}$. Figures 8(b) and 8(c) show that the fluid velocity $\overline{\left.u_{z}\right|_{C / C_{0}}}$ and the net force $\overline{\left.F_{z}\right|_{C / C_{0}}}$ exerted by the particles on the fluid have similar behavior, namely a pronounced decrease with increasing $C / C_{0}$. As $C / C_{0}$ becomes larger, the local but collective particle force accelerates the fluid along the gravitational direction, resulting in the enhancement of the downward velocity. This in turn significantly increases the settling velocity. Particles thus fall with the fluid surrounding them. Not shown here, the fluid velocity contribution increases with increasing $\Phi$. Note that the fluid acceleration contribution from pressure and viscous forces (not presented) was found to essentially oppose to particle back-reaction force such that the preferential sampling along fluid acceleration reported in our one-way coupling simulations is not observed any more in the two-way coupling simulations.

One can wonder on the robustness of the centrifuging mechanism [2] of heavy particles away from the highvorticity region when particles back-reaction force on the carrier phase is accounted for. Previously mentioned in Sec. III A, this mechanism, associated with particle preferential concentration and commonly observed maximal for $S t \sim 1$, and the related preferential sweeping of particles under gravity are invoked to explain the faster settling of particles in abscence of two-way coupling [2]. To figure out how far volume fraction and gravity alter the centrifuge mechanism, we present in Fig. 9 the joint PDFs of the fluid vorticity, $\Omega^{2}$, and fluid shear, $S^{2}$, defined by $\Omega_{i j}=\frac{1}{2}\left(\frac{\partial u_{i}}{\partial u_{j}}-\frac{\partial u_{j}}{\partial u_{i}}\right)$ and $S_{i j}=\frac{1}{2}\left(\frac{\partial u_{i}}{\partial u_{j}}+\frac{\partial u_{j}}{\partial u_{i}}\right)$, respectively, both computed at particle location for $S t=1$. As $\Omega^{2}$ and $S^{2}$ are widely distributed with very asymmetric PDFs, their mean values are not meaningful while the joint PDF representation provides a clear illustration of our purpose. Figure 9 shows that, in zero-gravity $(R=0)$, whatever the value of $\Phi$ considered, the most probable values of $S^{2}$ are twice those of $\Omega^{2}$, which in turn reflects persistence of the centrifuge effects. This is also featured for low values of the couple $R / \Phi$. However, as $R / \Phi$ further increase, most probable values deviate to large values of $\Omega^{2}$, meaning that the centrifuge effects are attenuated or even suppressed (see $\Phi=7 \times 10^{-5}$ and $R=1)$.

\section{SUMMARY AND CONCLUSIONS}

In this study, we have presented two-way coupling DNS of turbulent flow laden with heavy inertial particles at moderate Reynolds number in presence of gravity. We have quantified the modification of the settling velocity addressing the effects of particle inertia, gravity and particle volume fraction. We have analysed these effects on preferential concentration and fluid statistics (at particle position) to understand the mechanisms responsible for the observed alteration. We have presented as much as possible comparisons with the reference experimental work of Aliseda et al. [1] whose parameter space covers the one of the simulations. The qualitative (and often the quantitative) agreement between our simulations and experiments suggest that the minimal ingredient used in the 


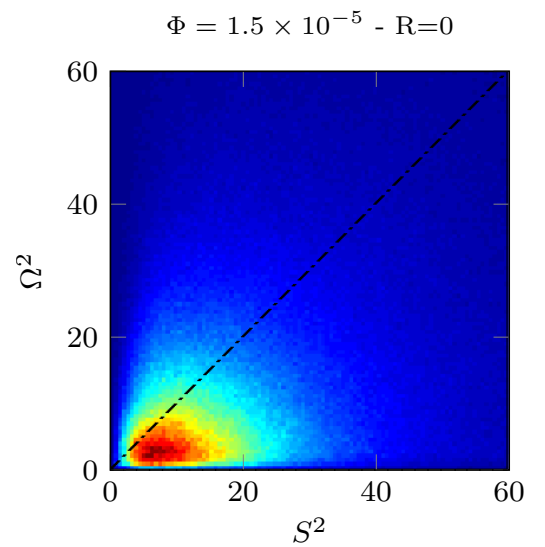

$\Phi=7 \times 10^{-5}-\mathrm{R}=0$

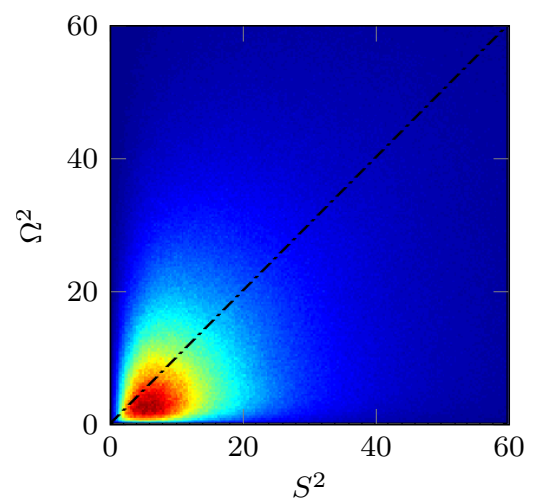

$\Phi=1.5 \times 10^{-5}-\mathrm{R}=0.25$

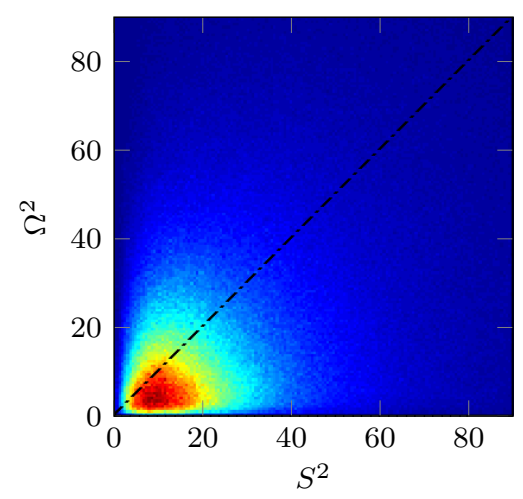

$\Phi=7 \times 10^{-5}-\mathrm{R}=0.25$

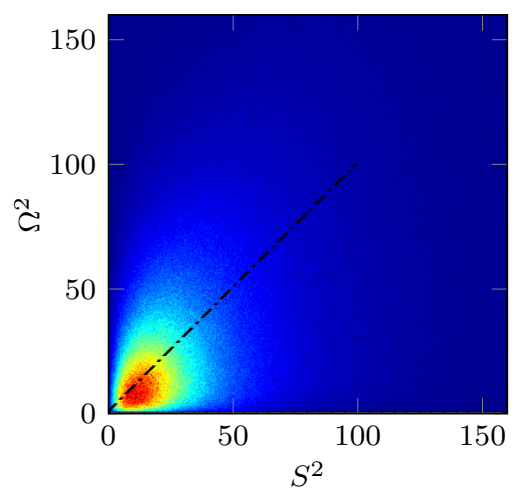

$\Phi=1.5 \times 10^{-5}-\mathrm{R}=1$

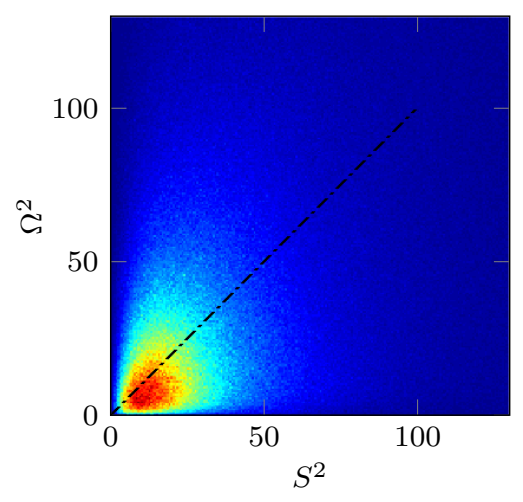

$\Phi=7 \times 10^{-5}-\mathrm{R}=1$

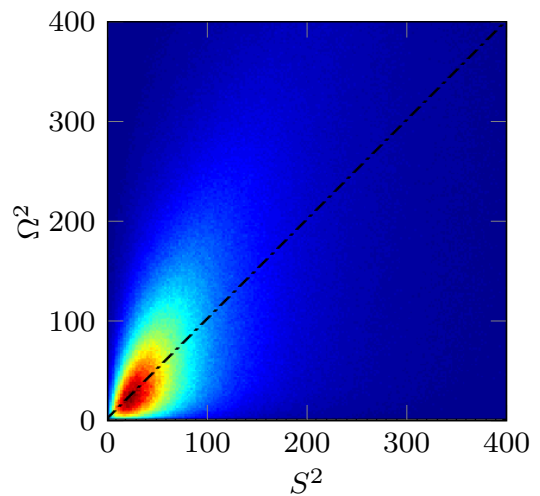

FIG. 9. Color online. Joint PDF of $S^{2}$ and $\Omega^{2}$ for $S t=1$. The black dash-dotted line is the first diagonal.

present DNS are enough to capture the physical mechanisms at work in actual turbulent flows laden with inertial particles at least with respect to settling velocity enhancement. The main conclusions follow.

The present study confirms that the settling velocity of particles falling in a homogeneous turbulent flow is further increased by momentum exchange between both phases, as previously reported in experiments [1] and DNS [4]. This is observed for all the examined Stokes numbers, ranging from 0.36 to 6 when based on the Kolmogorov time scale. At the considered volume fractions, the effects of two-way coupling are negligible on the overall statistics of turbulence. Nevertheless, the statistics conditioned on the local particle concentration reported here show that the collective force of particles accelerates, locally, the fluid and increases the fluid velocity surrounding the particles in the gravitational direction. The resulting modified fluid downward-velocity is identified as the main contribution of settling enhancement in presence of two-way coupling. Particles and fluid thus fall together, their relative velocity actually vanishing. This behavior is reminiscent of the model proposed by Aliseda et al. [1] in which groups of particles were considered as "meta-clusters" whose settling velocity is higher than the one of individual particles. In such a model, the slip velocity between fluid and particles should be very weak as we observe.

The maximum preferential concentration is still found for $S t$ around unity. The effects of gravity and particle volume fraction on $\sigma_{\mathcal{V}}$, analyzed for particles with $S t=1$, display a monotonous decrease of preferential concentration with increasing $R$, this decrease being stronger as $\Phi$ is larger. For a given $R$, a similar effect of $\Phi$ is observed for particles with $S t \leq 2$ while larger inertia particle exhibit a reverse tendency. The reduction of preferential concentration for $S t \sim 1$ is shown to be in relation with a local modification of the flow structure by the particles. In particular, a significant increase of vorticity is observed at particle position as $\Phi$ and/or $R$ increase. This leads to an attenuation or even suppression of centrifuge effects. Besides, for all the considered St, the preferential sampling of particles in downward fluid motion is observed to be amplified by the particle back-reaction force, as particles and fluid mutually entrain each other.

The anisotropy of the particle concentration field (previously observed in one-way coupling DNS $[7,30,33]$ ) is further increased under two-way-coupling. This manifests as a denser accumulation of particles in the downward direction with increasing $R$ and $\Phi$. It can explain the increase of preferential concentration observed for $S t=6$ in presence of momentum exchange, and also suggests a further reduction of the past-history effects 
along gravity compared to one-way coupling simulations [30].

The 2D Voronoï analysis of the particle field shows that cluster sizes are algebraically distributed with a power around -2 . This indicates the self-similar nature of preferential concentration in particle laden flows as already reported $[27,31,34]$. The cluster sizes distribution displays a peak within the small turbulence scales, consistently with former experimental observations [1]. The corresponding 3D analysis reveals a unique complex interconnected structure reminiscent of the results obtained by Calzavarini et al. [32]. The emergence of this structure deserves further investigations.

The reported local effects exerted by the collective force of particles on the fluid quantities (vorticity and shear) are expected to locally alter the turbulence scales.
The increase of particle field anisotropy under increasing $R$ and $\Phi$ can also be inferred to alter turbulence anisotropy (previously suggested in $[5,13,14]$ ). A scrutinized investigation of these local interaction requires a study apart accounting for effects of gravity and particle loading on turbulence scale and flow anisotropy related quantities.

\section{ACKNOWLEDGMENTS}

This study was partly supported by the Ramón y Cajal research program from the Spanish Ministry of Science. We thank the anonymous referee who suggested the present organisation of the paper.
[1] A. Aliseda, A. Cartellier, F. Hainaux, and J. C. Lasheras, "Effect of preferential concentration on the settling velocity of heavy particles in homogeneous isotropic turbulence," Journal of Fluid Mechanics 468, 77-105 (2002).

[2] L. P. Wang and M. R. Maxey, "Settling velocity and concentration distribution of heavy particles in homogeneous isotropic turbulence," Journal of Fluid Mechanics 256, 27-68 (1993).

[3] C. Y. Yang and U. Lei, "The role of the turbulent scales in the settling velocity of heavy particles in homogeneous isotropic turbulence," Journal of Fluid Mechanics 371, 179-205 (1998).

[4] T. Bosse, L. Kleiser, and Meiburg E., "Small particles in homogeneous turbulence: settling velocity enhancement by two-way coupling," Physics of Fluids 18, 027102 (2006).

[5] T. S. Yang and S. S. Shy, "Two-way interaction between solid particles and homogeneous air turbulence: particle settling rate and turbulence modification measurements," Journal of Fluid Mechanics 526, 171-216 (2005).

[6] G. H. Good, P. J. Ireland, Bewley G. P., Bodenschatz E., L. R. Collins, and Z. Warhaft, "Settling regimes of inertialparticles in isotropic turbulence," Journal of Fluid Mechanics 759, R3 (2014).

[7] A. Dejoan and R. Monchaux, "Preferential concentration and settling of heavy particles in homogeneous turbulence," Physics of Fluids 25, 013301 (2013).

[8] K. D. Squires and J. K. Eaton, "Particle response and turbulence modification in isotropic turbulence," Physics of Fluids A2, 1191-1203 (1990).

[9] M. Boivin, O. Simonin, and K. D. Squires, "Direct numerical simulation of turbulence modulation by particles in isotropic turbulence," Journal of Fluid Mechanics 375, 235-263 (1998).

[10] O. A. Druzhinin and S. Elghobashi, "Particle response and turbulence modification in isotropic turbulence," Physics of Fluids 11, 602-610 (1999).

[11] S. Sundaram and L. R. Collins, "A numerical study of the modulation of isotropic turbulence by suspended particles," Journal of Fluid Mechanics 379, 104-143 (1999).

[12] S. Elghobashi and G. C. Truesdell, "On the two-way interaction between homogeneous turbulence and dis- persed solid particles.I: turbulence modification," Physics of Fluids A5, 1790-1801 (1993).

[13] A. Ferrante and S. Elghobashi, "On the physical mechanisms of two-way coupling in particle-laden isotropic turbulence," Physics of Fluids 15, 315-329 (2003).

[14] A. Dejoan, "DNS experiments on the settling of heavy particles in homogeneous turbulence: two-way coupling and Reynolds number effects," Journal of Physics, Conference Series 333, 012006 (2011).

[15] M. Maxey and B. K. Patel, "Localized force representations for particles sedimenting in Stokes flow," International Journal of Multiphase Flow 27, 1603-1626 (2001).

[16] C. T. Crowe, "Review-numerical methods for dilute gasparticle flows," Journal of Fluid Engineering 104 (3), 297 (1982).

[17] M. R. Maxey and J. J. Riley, "Equation of motion for a small rigid sphere in a nonuniform flow," Physics of Fluids 26, 883-889 (1983).

[18] R. Gatignol, "The Faxen formulae for a rigid particle in an unsteady non-uniform Stokes flow," Journal de Mécanique Théorique et Appliquée 2, 143-160 (1983).

[19] R. Rogallo, "Numerical experiments in homogeneous turbulence," NASA Technical Report (1981).

[20] J. Jímenez and A.A. Wray, "On the characteristics of vortex filaments in isotropic turbulence," Journal of Fluid Mechanics 373, 255-285 (1998).

[21] S. Elghobashi, "An updated classification map of particle-laden flows," in Proc. IUTAM Symposium on Computational Multiphase Flows, Springer (2006) pp. 210.

[22] E. J. P. Woittiez, H. J. J. Jonker, and L. M. Portela, "On the combined effects of turbulence and gravity on droplet collisions in clouds: a numerical study," Journal of the Atmospheric Sciences 66, 1926-1943 (2009).

[23] K. D. Squires and J. K. Eaton, "Particle response and turbulence modification in isotropic turbulence," Physics of Fluids 2, 1191-1203 (1990).

[24] C. Poelma, J. Westerweel, and G. Ooms, "Particlefluid interactions in grid-generated turbulence," Journal of Fluid Mechanics 589, 315-351 (2007).

[25] R. Monchaux, M. Bourgoin, and A. Cartellier, "Analyzing preferential concentration and clustering of inertial 
particles in turbulence," International Journal of Multiphase Flow 40, 1-18 (2012).

[26] R. Monchaux, "Measuring concentration with Voronoï diagrams: the study of possible biases," New Journal of Physics 14, 095013 (2012).

[27] R. Monchaux, M. Bourgoin, and A. Cartellier, "Preferential concentration of heavy particles: A Voronoi analysis," Physics of Fluids 22, 103304 (2010).

[28] S. Sumbekova, A. Cartellier, A. Aliseda, and M. Bourgoin, "Preferential concentration of inertial subKolmogorov particle. The roles of Reynolds numbers," Physical Review Fluids 2, 024302 (2017).

[29] J. Bec, H. Homann, and S. S. Ray, "Gravity-DRiven Enhancement of Heavy Particle Clustering in turbulent Flow," Physical Review E 112(18), 184501 (2014).

[30] P. J. Ireland, A. D. Bragg, and L. R. Collins, "The ef- fetcs of Reynolds number on inertial particle dynamics in isotropic turbulence. Part II: Simulations with gravitational effects," Journal of Fluid Mechanics 796, 659-711 (2016).

[31] H. Yoshimoto and S. Goto, "Self-similar clustering of inertial particles in homogeneous turbulence," Journal of Fluid Mechanics 577, 275-+ (2007).

[32] E. Calzavarini, M. Kerscher, D. Lohse, and F. Toschi, "Dimensionality and morphology of particle and bubble clusters in turbulent flow," Journal of Fluid Mechanics 607, 13-24 (2008).

[33] J. Bec, H.. Homann, and S. S. Ray, "Gravity-Driven enhancement of heavy particle clustering in turbulent flow," Physical Review Letter 112, 184501 (2014).

[34] G. Boffetta, F. de Lillo, and A. Gamba, "Large scale inhomogeneity of inertial particles in turbulent flows," Physics of Fluids 16, L20-L23 (2004). 